\title{
Assessment of visual demand of typical data entry tasks in automotive navigation systems for iPhone
}

\author{
Manuela Quaresma ${ }^{\mathrm{a}}$ \\ ${ }^{a}$ LEUI Laboratory of Ergonomics and Usability of Interfaces in Human-Technology Systems, PUC-Rio Pontifical \\ Catholic University of Rio de Janeiro, Rua Marquês de São Vicente, 225 - 713F, 22451-900 Rio de Janeiro/RJ, \\ Brazil, Tel.+55 (21) 3527 1005, Fax.+55 (21)3527 1589, info@manuelaquaresma.com
}

\begin{abstract}
This paper aims to present an assessment of the visual demand of typical data entry tasks in two popular navigation systems among iPhone Brazilians users - TomTom App and iGO Primo App. Applying the extended KLM technique, the total times to perform the tasks with interruptions were estimated, with which it was possible to evaluate the potential for driver distraction as per criteria established by some recommended practices of automotive industry. With the results obtained, it was identified that the main issues that lead to increased visual demand afforded by the systems are related to the amount of screens that are required in the process of data entry and the failure to implement iOS features in applications developed for it.
\end{abstract}

Keywords: interface design, usability, navigation systems, driver distraction, task analysis

\section{Introduction}

The advancement of computer technology in vehicle systems has been quite intense in the last years. In general, there are two kinds of in-vehicles systems: the advanced driver assistance systems (ADAS) and the in-vehicle information systems (IVIS). Among the IVIS, the navigation systems are some of the most popular in the Brazilian market.

Even though the automotive navigation systems emerged a few decades ago, they only had a wide spread use in the last five years, primarily in Brazil. Much of this spread is related to the evolution of digital technologies, such as LCD color displays, and the cheapening of portable devices.

Nowadays, navigation systems run on four types of devices: on portable GPS units (hardware dedicated to GPS navigation mounted on brackets on vehicles windshield or dashboard), on vehicle original factory equipments (in-dash navigation systems), on aftermarket equipments (devices that can be integrated into the DIN slot for radio/CD) and on Smartphones (that have GPS antenna). However, this latter type of device has promoted the popularity of these systems in a much more extensive way and appears to be a tendency to the use of GPS systems.

\section{Problem}

Although the use of these systems can bring benefits, they must be carefully designed to avoid distractions to the driver in his primary task - driving the vehicle safely. If the interface design of the systems does not consider the usability principles and criteria, they certainly have a great potential to distract the driver while driving the vehicle [11].

According to Lee et al [9] the driver distraction is defined as "a diversion of attention away from activities critical for safe driving toward a competing activity". However, as the distraction occurs by several sources it is important to understand it in terms of four categories: visual distraction (e.g., looking away from the roadway), auditory distraction (e.g., responding to a ring cell phone), biomechanical distraction (e.g., manually adjusting the radio volume) and cognitive distraction (e.g., being lost in thought) [14]. 
Considering that the driving task requires the driver's attention to what is happening in front of him, the visual distraction can be considered the category that most occurs, and consequently, the one that can cause more accidents. Wang et al apud Burns [4] stated that $13 \%$ of accidents with vehicles in US were related to visual distraction and part of this could be related to the use of IVIS. Brooks [3] also states that the higher the system's visual demand the more dangerous the driving task.

\section{Method}

In order to evaluate the potential visual distraction of automotive navigation systems developed for iPhone, primarily related to visual demand afforded by the systems, the total time to perform typical data entry tasks was estimated by applying the Extended KLM technique, proposed by Pettitt [12].

The Extended KLM technique is based on the simplest variant of GOMS model, the well-known KLM (Keystroke Level Model) [5], which is a analytical evaluation model that estimates the time of a task carried out by an experienced user, and is also based on the occlusion technique, established by ISO 16673 [7], in order to predict the total time of the task performed with interruptions. ISO 16673 [7] defines the occlusion technique as a "measurement method involving periodic obstruction of participant's vision or the obscuration of visual information under investigation." This international standard was developed to "provides a procedure for measuring visual demand due to the use of visual or visual-manual interfaces accessible to the driver while the vehicle is in motion", applying to both Original Equipment Manufacturer (OEM) and aftermarket in-vehicle system.

The primary objective of the occlusion technique is to assess the visual demand afforded by IVIS, as well as simulate the interruption of a task performed in the system. This technique is based on the assumption that the driver while driving using an IVIS must balance his vision, alternating between looking the road and reading the system display and controls. From this, in order to simulate normal driving conditions, the technique uses a device that blocks the vision of participants (such as PLATO - portable liquid-crystal apparatus for tachistoscopic occlusion) [6] at certain time intervals, varying between occlusion and vision intervals at the moment that the participant performs a given task in the IVIS investigated, with the vehicle parked. The occlusion interval simu- lates the time when the driver would be looking at the road, while the vision interval simulates the time when the driver would be performing a task in the system. ISO 16673 establishes the duration of 1.5second for both intervals of occlusion and vision.

The main parameters provided by the occlusion technique are: the 'total task time under unoccluded conditions' $\left(\mathrm{TTT}_{\text {unoccl }}\right)$, the 'total shutter open time' (TSOT) and 'resumability' index (R). The 'TTT unoccl' refers to the total time required to perform a task without interruptions, while the 'TSOT' is the total duration of all intervals of vision while performing the task with interruptions and is related to the time that the driver would not be looking at the road. ' $\mathrm{R}$ ' is the ratio of the duration of 'TSOT' and 'TTT ${ }_{\text {unoccl }}$ (i.e., TSOT / $\mathrm{TTT}_{\text {unoccl }}$ ), which means how easy a task can be resumed after several interruptions. Values of ' $R$ ' less than 1 indicate that the task might be performed even at intervals of occlusion - a task that requires less visual demand to be completed.

In the same way as is carried out in model GOMSKLM, to apply the Extended KLM technique the researcher first decomposes the task into sequences of operators (physical and mental actions), considering the user's goals, methods and selections rules to complete a given task, setting the value of $\mathrm{TTT}_{\text {unoccl }}$ (total task time under unoccluded conditions). Once the decomposition is accomplished, the researcher distributes the operators along intervals of vision and occlusion in the same way the task is "partitioned" in the occlusion technique. Figure 1 shows an example of the distribution of operators along vision/occlusion intervals. As a result, the estimated values are obtained for the TSOT (total shutter open time) and, consequently, for the R-value ('resumability' index). To distribute the operators along the sequence of intervals of vision and occlusion, the author [12] established three assumptions that define when an operator (action) can occur at occlusion intervals, thus enabling the evaluation of tasks that may be visually interrupted or not.

For the development of interfaces for IVIS, in order to compare design solutions, the Extended KLM is quite useful, simple and easy to be applied to assess the visual demand afforded by IVIS. With this model, it is possible to evaluate, since the beginning of the development of design solutions, if the new HMI (human-machine interface) will meet to criteria of task time performance while the vehicle is in motion, like the 20 seconds for TSOT of SAE J2364 (vision interrupted method) [15] and AAM (principle 2.1) [1]. 


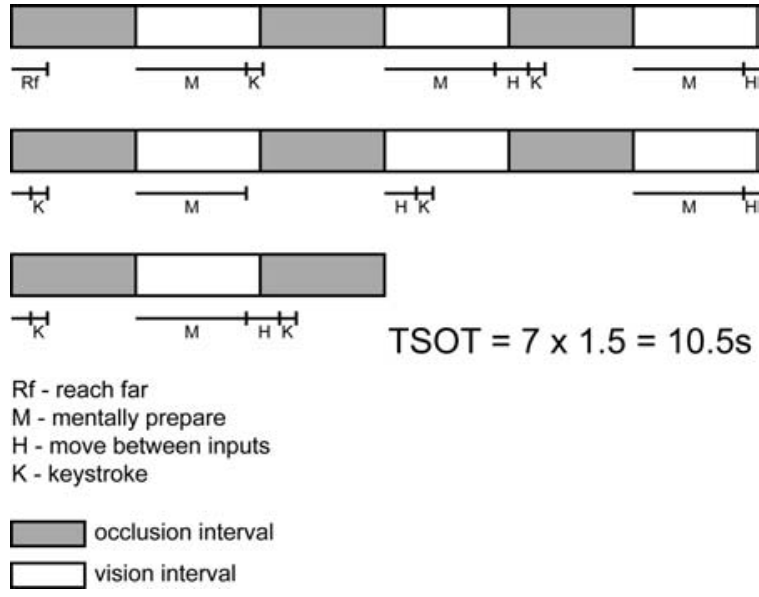

Fig. 1 - Example of Extended KLM modeling

\subsection{Systems}

The navigation systems evaluated were: Tomtom Brasil App Version 1.8 and Brasil - iGO Primo Version 2.0.5 App, both running on iPhone 4, iOS 4.3.5. Also, both systems were in the Top 10 chart in Brazilian App Store during the study.

\subsection{Tasks}

Three common, comparable tasks on both systems were selected for assessment. These were:

- Task 1 - set a destination by address;

- Task 2 - select as a destination the gas station (POI - Point of Interest) closest to the current position;

- Task 3 - store a new home address (favorite address).

The addresses used in the first and third tasks were the same for all systems.

Applying the Extended KLM technique, in the first stage the tasks were decomposed in sequences of operators, considering the task goals and subgoals, the methods and selection rules, following the guidelines of KLM model. The methods and selection rules used were based on a previously usability testing conducted on similar systems (PDA versions of the same systems) and for the same tasks [13]. In the second stage, the operators were distributed along the sequence of intervals of vision and occlusion (1.5- seconds per interval), as shown on Figure 1, considering the assumptions established by [12].

\section{Results}

The tables 1, 2 and 3 demonstrate the estimated total time to perform the three tasks, respectively, on the systems assessed, under unoccluded conditions ( $\left.\mathrm{TTT}_{\text {unoccl }}\right)$ and with interruptions (TSOT), as well as the R-value (resumability), resulting from the Extended KLM modeling process. It is observed that only on TomTom App all tasks can be performed in less than 20 seconds (criterion for TSOT [1] [15]) and just in the Task 2 the iGO Primo App meet the 20 seconds criterion. Also, all Rvalues obtained are quite reasonable in all the tasks ( $R$ $<1$ ), especially for task 2 , which means that the interfaces design solutions are appropriate for performing tasks with interruptions.

In task 1 , one of the main reasons that led the iGO Primo App take more time was one more screen in the data entry process to confirm the address. In this system, after the entry of the city name, street name and building number, the user must confirm the address data (Figure 2 ) in order that the system calculate the route and then he confirms the route planned (Figure 3), whereas on TomTom App after the address entry (city, street and number), the system immediately calculates the route, presenting a summary screen of the data and the calculated route (Figure 4) to be confirmed. There is no reason for the additional screen (for address data confirmation) in the process of setting the destination, except for the use of other features in the system (iGO Primo App), however as this task is the most frequently carried out on automotive navigation systems, the execution time and simplification of the task should be prioritized.

Table 1

Results of Task 1 (set a destination by address)

\begin{tabular}{cccc} 
& \multicolumn{3}{c}{ TASK 1 } \\
\cline { 2 - 4 } & TTT $_{\text {unocl }}$ & TSOT & R \\
\hline TomTom App & $19.25 \mathrm{~s}$ & $18.0 \mathrm{~s}$ & 0.93 \\
\hline iGO Primo App & $22.55 \mathrm{~s}$ & $21.0 \mathrm{~s}$ & 0.93 \\
\hline $\begin{array}{l}\text { Systems that meet the } \\
\text { 20s criterion [1] [15] }\end{array}$ & & TomTom App & \\
\hline
\end{tabular}




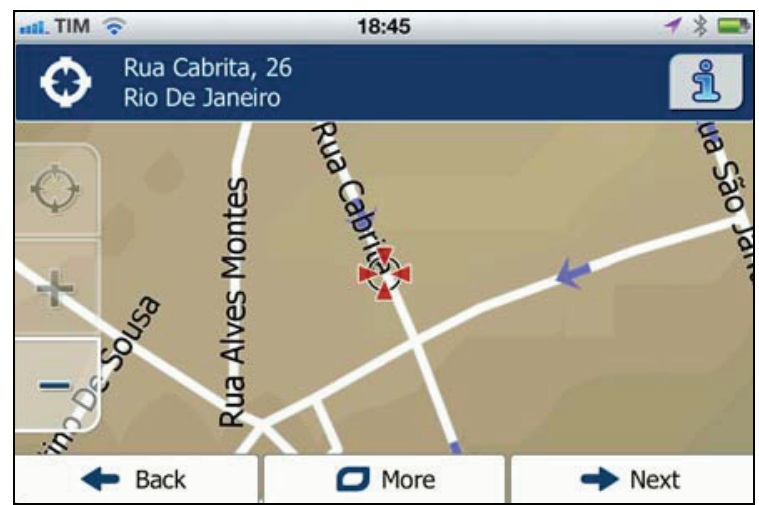

Fig. 2 - Screen for address data confirmation (iGO Primo App)

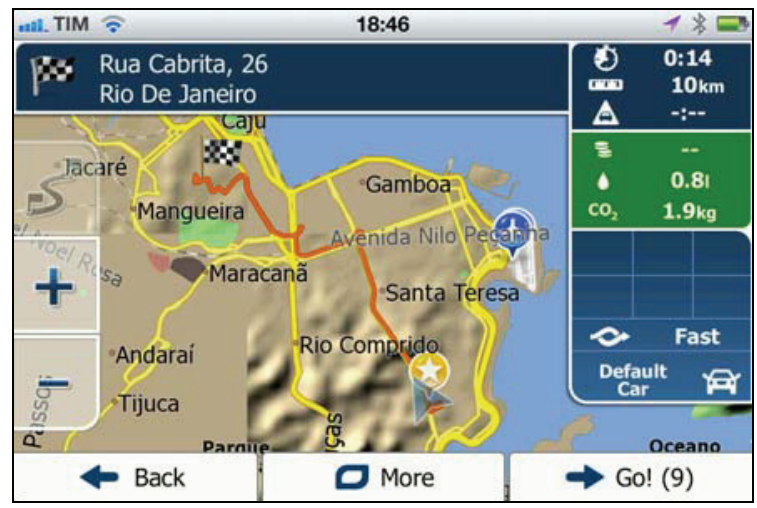

Fig. 3 - Screen for route confirmation (iGO Primo App)

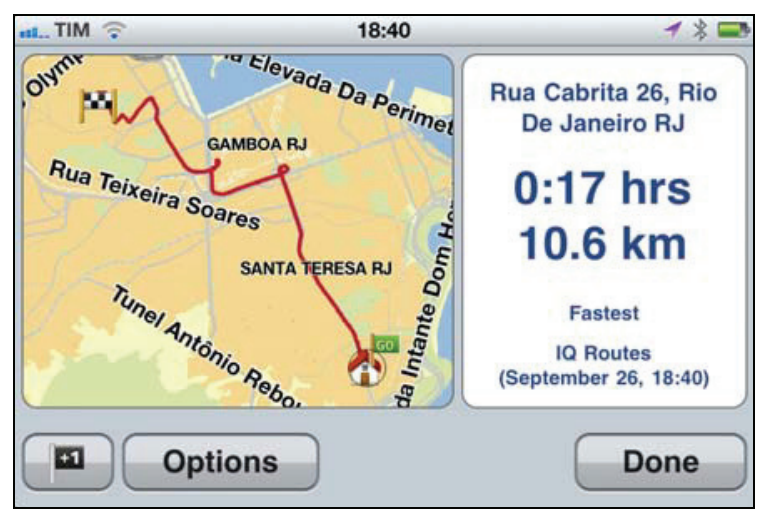

Fig. 4 - Screen for address and route confirmation (TomTom App)

In task 2, iGO Primo App had a better result in the estimated time to perform the task. The main fact was due to the combination of two steps in the selection process of point of interest - the step of selecting the reference of proximity (distance of the POI relative to a given point) and the step of selecting a POI category (Figure 5). On TomTom App it is needed first select the reference of proximity (Figure 6) and then select the desired category (Figure 7).

The iGO Primo App assumes that the reference of proximity 'Around Here' is the most frequently used for this kind of POI, which is probably true, since the gas station is a service used in times of immediate need. Also, it is important to note that both systems give priority to gas station POI category, because on the two systems it is always displayed in the first place. This greatly speeds up user interaction and allows the POI selection task be faster, primarily because it is a task performed with quite frequency while the vehicle is in motion. Prioritize the categories of POI is the best solution for the POI selection as a destination, instead of presenting long lists of categories [13]

\section{Table 2}

Results of Task 2 (select as a destination the gas station (POI) closest to the current position)

\begin{tabular}{cccc}
\cline { 2 - 4 } & \multicolumn{3}{c}{ TASK 2 } \\
\cline { 2 - 4 } TomTom App & $16.25 \mathrm{~s}$ & $12.0 \mathrm{~s}$ & 0.74 \\
\hline TT & unocl & TSOT & R \\
\hline iGO Primo App & $11.75 \mathrm{~s}$ & $9.0 \mathrm{~s}$ & 0.76 \\
\hline $\begin{array}{l}\text { Systems that meet the } \\
\text { 20s criterion [1] [15] }\end{array}$ & TomTom App and iGO Primo App \\
\hline
\end{tabular}

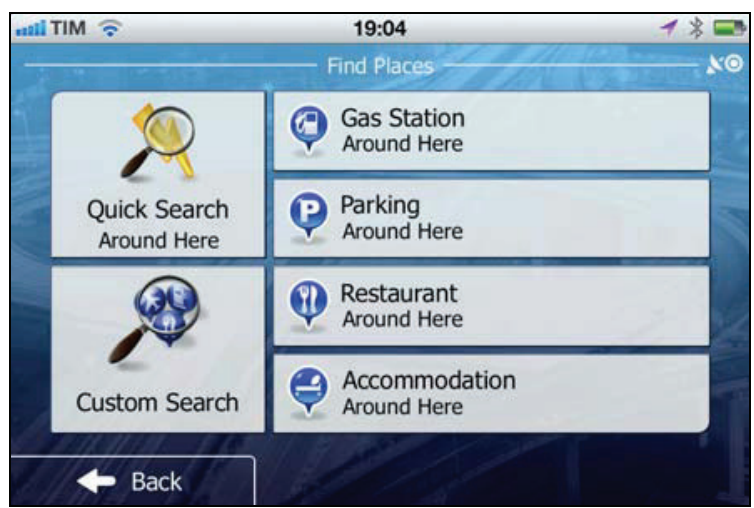

Fig. 5 - Screen to select the POI category and the reference of proximity (iGO Primo App) 


\begin{tabular}{|c|c|c|}
\hline mall. TIM ร & 18:58 & $1 \geqslant 0$ \\
\hline Navigate To... & Point of Interest & \\
\hline & POI Near Me & $>$ \\
\hline & POI in City & $>$ \\
\hline & POI Near Home & $>$ \\
\hline
\end{tabular}

Fig. 6 - Screen to select the POI reference of proximity (TomTom App)

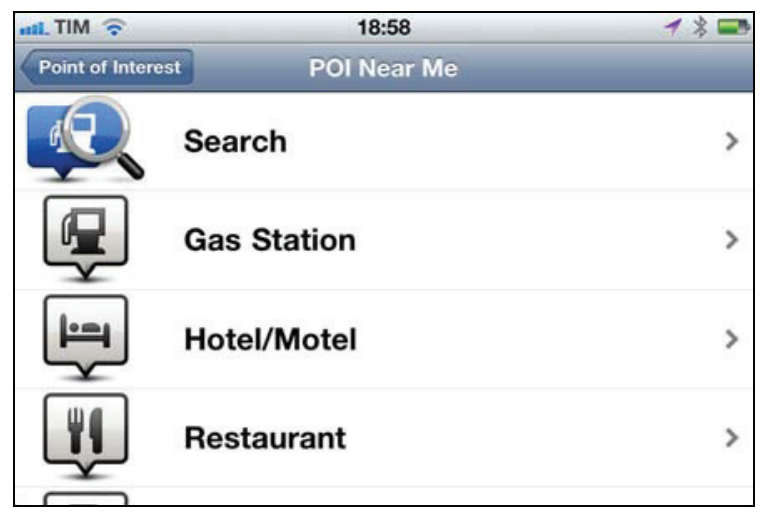

Fig. 7 - Screen to select the POI category (TomTom App)

In Task 3, although it is not a task performed frequently while the vehicle is in motion, the TomTom App meets the criterion of 20 seconds. The main reasons were: the use of operating system features, and the dedicated place for the favorite home. On TomTom App, all menu options use the scrolling feature (move the finger up or down across touch-screen surface) of iOS. Using the iPhone's scrolling feature makes the system structure less deep and therefore it makes that user does not need to navigate through many screens as in systems without scrolling. To store the home favorite address on iGO Primo App the user needs to navigate through 10 different screens to complete the task, while on TomTom App the task is completed through eight screens. In addition, the scrolling feature allows actions to be performed at intervals of occlusion, because the user does not need to look while he drags his finger across the screen when there are lists of options at known positions.

In iGO Primo App, the storing process of a favorite is only done after the entry of an address and there is no dedicated option for the favorite home, unlike the process on TomTom App. Besides taking more time to perform the task, iGO App Primo presents a solution that might be more confusing to the user.

Table 3

Results of Task 3 (store a new home address)

\begin{tabular}{cccc}
\cline { 2 - 4 } & \multicolumn{3}{c}{ TASK 3 } \\
\cline { 2 - 4 } TomTom App & TT $_{\text {unocel }}$ & TSOT & R \\
\hline \multirow{2}{*}{ iGO Primo App } & $21.80 \mathrm{~s}$ & $19.5 .0 \mathrm{~s}$ & 0.89 \\
\hline $\begin{array}{l}\text { Systems that meet the } \\
\text { 20s criterion [1] [15] }\end{array}$ & & TomTom App & \\
\hline
\end{tabular}

\section{Conclusion}

According to the results obtained in this study, it is possible to conclude that visual demand afforded by automotive navigation systems is directly related to the amount of screens that should be read in the data entry process. The more screens are required to complete a task, more attention the user will have to spend with the system and the greater are the chances of driver distraction. With the examples that were seen, there are solutions that can minimize the data entry processes. The best solutions should always be applied in the design of IVIS interfaces that will be used by drivers, when time is critical. Furthermore, it is important that the operating system features be implemented in the system's applications, because aside from the reduction of the time for user interaction, it also meets the usability principle of 'compatibility' proposed by Nielsen [10], Bastien and Scapin [2] and Jordan [8] - the way the interaction is compatible with user expectations.

Although the Extended KLM requires careful attention in defining the operators and how to distribute them along intervals of vision and occlusion, once learned it can be readily applied in the assessment of various tasks performed on IVIS. The important point of this technique is that it can be applied early in the development of HMIs, when certain design solutions can be compared. Moreover, when the technique is associated with other usability evaluation methods, like heuristic evalua- 
tion and usability testing, it can generate very rich results for choosing the better interface alternatives and interactions.

\section{References}

[1] AAM. Statement of principles, criteria and verification procedures on driver interactions with advanced in-vehicle information and communication systems. Washington, D.C.: Alliance of Automobile Manufacturers, 2007.

[2] Bastien, J.M.C. \& Scapin, D. Ergonomic Criteria for the Evaluation of Human Computer Interfaces. Le Chesnay: INRIA, 1993.

[3] Brooks, C.; Rakotonirainy, A.. In-vehicle technologies, advanced driver assistance systems and driver distraction: research challenges. In: Proceedings of International Conference on Driver Distraction, Sydney, 2005.

[4] Burns, P.; Ladsdown, T.. E-distraction: the challenges for safe and usable Internet services in vehicle. In: Proceedings of NHTSA Driver Distraction Internet Forum. Rockville: Westat, 2000.

[5] Card, S. K.; Moran, T.P.; Newell, A. The Keystroke-Level Model for User Performance Time with Interactive Systems. Communications of the ACM, 1980.

[6] Gelau, C.; Krems, J.F. (eds) The occlusion technique. Applied Ergonomics, London, 2004, v.35, n.3, p. 185-310.

[7] ISO. Road vehicles -- Ergonomic aspects of transport information and control systems -- Occlusion method to assess visual demand due to the use of in-vehicle systems. Génève: International Organization Standardization, 2007.

[8] Jordan, Patrick W.. An introduction to usability. London: Taylor \& Francis, 1998.

[9] Lee, J.D.; Young, K.L., Regan, M.A.. Defining driver distraction. In: Regan, M.; Lee, J.D.; Young, K. (eds). Driver distraction: theory, effects and mitigation. Boca Raton: CRC Press, 2009, p. 31-40.

[10]Nielsen, Jakob; Mack, R. L.. Usability inspection methods. New York: John Wiley \& Sons, 1994.

[11] Nowakowski, C.; Green, P.; Tsimhoni, O.. Common automotive navigation system usability problems and a standard test protocol to identify them. In: Proceedings of ITS-America 2003 Annual Meeting. Washington DC: Intelligent Transportation Society of America, 2003.

[12] Pettitt, M.. Visual demand evaluation methods for in-vehicle interfaces. Nottingham: University of Nottingham, Thesis Human-Computer Interaction, 2008.

[13]Quaresma, M.; Moraes, A.. Avaliação da usabilidade de sistemas de informação disponíveis em automóveis: um estudo ergonômico de sistemas de navageção GPS. Rio de Janeiro: PUC-Rio. 340p. Thesis - Arts and Design Department, 2010.

[14] Ranney, T. et al.. NHTSA Driver Distraction Research: past, present, and future. In: Proceedings of NHTSA Driver Distraction Internet Forum. Rockville: Westat, 2000.

[15] SAE. Navigation and route guidance function accessibility while driving. (SAE Recommended Practice J2364). Warrendale: SAE International, 2004. 